Chirurg 2014 · 85:821

DOI 10.1007/s00104-014-2857-0

Online publiziert: 13. August 2014

c) Springer-Verlag Berlin Heidelberg 2014

H. Dralle

Universitätsklinik für Allgemein-, Viszeral- und Gefäßchirurgie, Medizinische Fakultät,

Universitätsklinikum Halle, Halle (Saale)

\title{
Chirurgische Besonderheiten kleiner Nebenschilddrüsenadenome
}

tens $50 \%$ verbunden mit dem Erreichen des Normalbereiches.

\section{Ergebnisse}

The small abnormal parathyroid gland is increasingly common and heralds operative complexity. World J Surg 38:1274-1281

\section{Hintergrund und Fragestellung}

Die routinemäßige Serumkalziumbestimmung hat zu einer früheren Erkennung des primären Hyperparathyreoidismus (pHPT) geführt. Das Symptomspektrum hat sich darunter verändert. Während früher bei pHPT-Patienten Nephrolithiasis und Knochenerkrankungen dominierten, sind die vorherrschenden Symptome heute bei Patienten mit nur gering erhöhtem Serumkalzium und Parathormon vor allem unerklärte Müdigkeit, kognitive und Schlafstörungen. Ziel der vorliegenden Studie war daher, herausfinden, ob und wenn ja welche chirurgisch relevanten Besonderheiten kleine Nebenschilddrüsenadenome (NSDA) mit niedrig erhöhten Serumkalzium- und Parathormonwerten aufweisen.

\section{Methoden}

Die retrospektive Untersuchung umfasste insgesamt 3974 pHPT-Patienten, die 1997 bis 2011 in einem universitären Zentrum von vier endokrinen Chirurgen unter Einsatz der intraoperativen PTH-Bestimmung (IOPTH) parathyreoidektomiert wurden. Bei allen Patienten erfolgte präoperativ eine bildgebende Lokalisationsdiagnostik und intraoperativ eine Größen- und Gewichtsbestimmung der entfernten Nebenschilddrüse(n) (NSD). Bei einem Gewicht von $<50 \mathrm{mg}$ wurde die NSD als normal gewertet, bei einem Gewicht von bis zu 200 mg als Mikroadenom. Erfolgskriterium einer erfolgreichen Parathyreoidektomie (PTX) war der intraoperative $\mathrm{PTH}-\mathrm{Abfall}$ von mindes-

\section{Diskussion und Fazit}

Die infolge routinemäßiger Serumkalziumbestimmung zunehmende Früherkennung des pHPT hat zur Folge, dass vermehrt NSDA geringerer Größe vorliegen und die Rate präoperativ negativer Bildgebungen steigt. Chirurgisch relevantes Hauptergebnis der Studie ist, dass bei initial im Rahmen der Exploration resezierten Mikroadenomen die Häufigkeit von Mehrdrüsenerkrankungen und das Misserfolgsrisikos signifikant ansteigen. Für die chirurgische Praxis folgt daraus folgendes Fazit:

- Bei eindeutiger Laborchemie eines pHPT besteht trotz der bei Mikroadenomen mit niedrig erhöhten Kalziumund Parathormonwerten häufig negativen Bildgebung wegen der fast immer eruierbaren Symptome in aller Regel eine Operationsindikation mit Aussicht auf erfolgreiche Beseitigung des pHPT.

- Bei Mikroadenomverdacht (niedrig erhöhtes Serumkalzium und Parathormon, negative Bildgebung) sollte primär, spätestens jedoch bei inadäquatem Abfall des IOPTH nach der erstresezierten NSD eine Vierdrüsenexploration erfolgen. Um Misserfolge zu vermeiden, sollten NSD-Operationen bei Mikroadenomen daher nur bei Verfügbarkeit und strengen Erfolgskriterien des IOPTH vorgenommen werden.

\section{Korrespondenzadresse}

Prof. Dr. Dr. h.c. H. Dralle

Universitätsklinik für Allgemein-, Viszeralund Gefäßchirurgie, Medizinische Fakultät, Universitätsklinikum Halle, Ernst-Grube-Str. 40, 06097 Halle (Saale) henning.dralle@uk-halle.de

Interessenkonflikt. H. Dralle gibt an, dass kein Interessenkonflikt besteht. 